\title{
Tangible User Interfaces Compensate for Low Spatial Cognition
}

\author{
John Quarles* \\ Dept. of CISE \\ University of Florida
}

\author{
Samsun Lampotang †े \\ Dept. of Anesthesiology \\ University of Florida
}

\author{
Ira Fischler: \\ Dept. of Psychology \\ University of Florida
}

\author{
Paul Fishwick* \\ Dept. of CISE \\ University of Florida
}

\author{
Benjamin Lok* \\ Dept. of CISE \\ University of Florida
}

\begin{abstract}
This research investigates how interacting with Tangible User Interfaces (TUIs) affects spatial cognition. To study the impact of TUIs, a between subjects study was conducted $(n=60)$ in which students learned about the operation of an anesthesia machine. A TUI was compared to two other interfaces commonly used in anesthesia education: (1) a Graphical User Interface (a 2D abstract simulation model of an anesthesia machine) and (2) a Physical User Interface (a real world anesthesia machine). Overall, the TUI was found to significantly compensate for low user spatial cognition in the domain of anesthesia machine training.
\end{abstract}

KeYwords: Tangible Interfaces, Mixed Reality, Spatial Cognition, Anesthesia Machine, User Studies

IndeX TeRms: J.3 [Computer Applications]: Life and Medical Sciences - Health;

\section{INTRODUCTION}

Spatial cognition deals with how humans encode spatial information (i.e. about the position, orientation and movement of objects in the environment), and how this information is represented in memory and manipulated internally [5]. This research investigates how spatial cognition may be affected by Tangible User Interfaces (TUIs). In previous work, we showed that a TUI enabled students to more effectively learn how their physical interactions with an anesthesia machine controlled internal machine function [16]. These previous findings identified the anesthesia training application as a useful vehicle for exploring how spatial cognition may be facilitated by a TUI.

To investigate the impact of TUI on spatial cognition, a between-subjects study was conducted with sixty participants. This study was part of a larger investigation into how mixed reality can enhance learning of complex concepts (i.e. anesthesia machine concepts). In the study presented in this paper, participants were trained in the operation of an anesthesia machine by training with one of three interfaces (figure 1) (20 participants in each condition):

(1) Physical User Interface (PUI) - The Anesthesia Machine (AM) itself.

(2) Graphical User Interface (GUI) The Virtual Anesthesia Machine (VAM) - a desktop-based 2D abstract simulation.

(3) Tangible User Interface (TUI) - The Augmented Anesthesia Machine (AAM) - a 3D abstract simulation of an AM that employs: the physical AM as a TUI, a geometric model of the AM, and a 6DOF magic lens.

* email: \{jpq, fishwick, lok\}@cise.ufl.edu

$\uparrow$ email: SLampotang@anest.ufl.edu

† email: ifisch@ufl.edu

IEEE Symposium on 3D User Interfaces 2008 8-9 March, Reno, Nevada, USA

978-1-4244-1972-2/08/\$25.00 @2008 IEEE

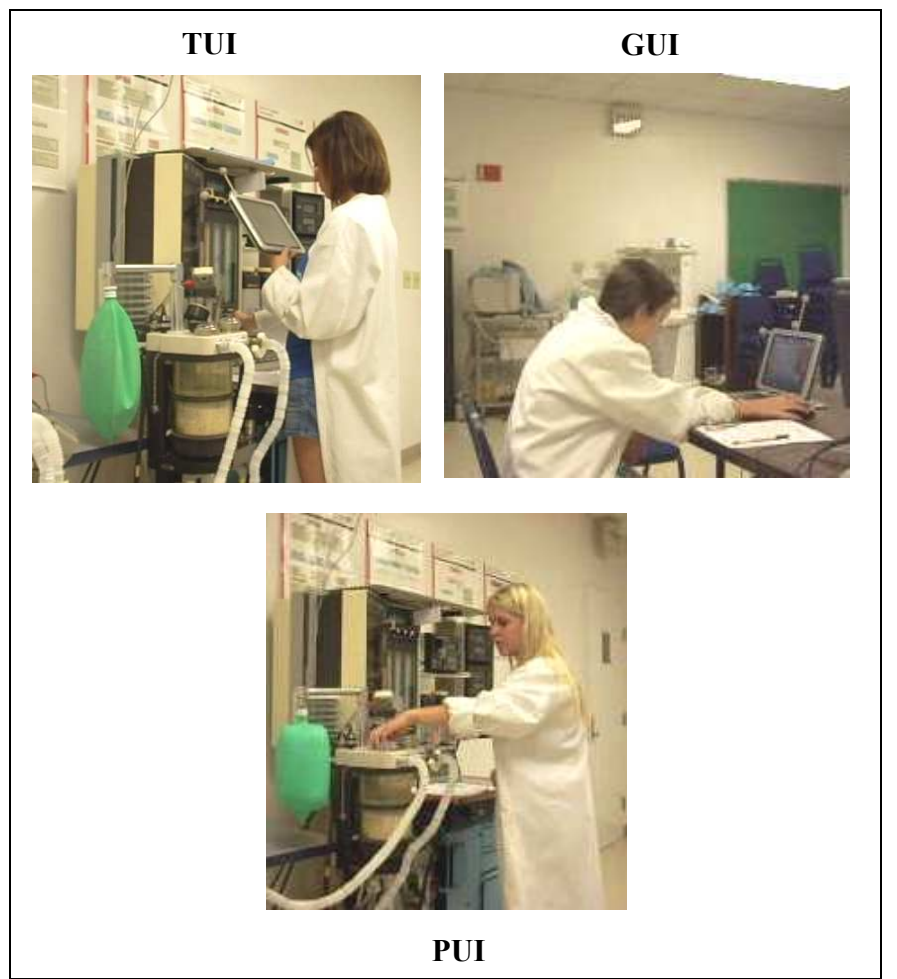

Figure 1. A study was conducted to investigate how three interface types (GUI, PUI, TUI) affect spatial cognition differently.

Note that the GUI and the PUI used in this study are widely used interfaces in anesthesia education. In comparing the GUI, the PUI, and the TUI, we explored how these three interfaces might facilitate spatial cognition differently. Through this comparison, we attempt to identify the spatial cognitive benefits unique to TUIs.

Specifically, through this anesthesia machine research, our research identifies generalizable findings related to:

- The types of spatial challenges for which TUIs are most beneficial

- The populations that experience the most spatial cognitive benefits from TUIs

- $\quad$ Methods to identify users that benefit from TUIs

\section{Motivating Application}

To study how TUIs affect spatial cognition, this research uses the domain of anesthesia machine training. Understanding and operating an anesthesia machine involves solving many problems related to spatial cognition (i.e. mentally visualizing invisible gas flow in the context of the anesthesia machine). Currently, some students first train with the Virtual Anesthesia Machine (VAM) (figure 2), a 2D abstract, transparent reality simulation [8] of an anesthesia machine. One of the advantages (and disadvantages) of the VAM is that its spatial organization is simpler than a physical 
machine. This simplification makes abstract concepts (such as gas flow) easier to visualize, follow, understand and retain.

After students practice with the VAM, they move on to practice with a real anesthesia machine. The actual anesthesia machine allows students to learn the procedural concepts (i.e. psychomotor skills, how to physically interact with the machine). However, some students have difficulty transferring and applying the VAM's abstract concepts when interacting with the physical machine. It was hypothesized that students encounter this problem because they have difficulty spatially mapping the simplified VAM layout to the more complex physical machine layout.

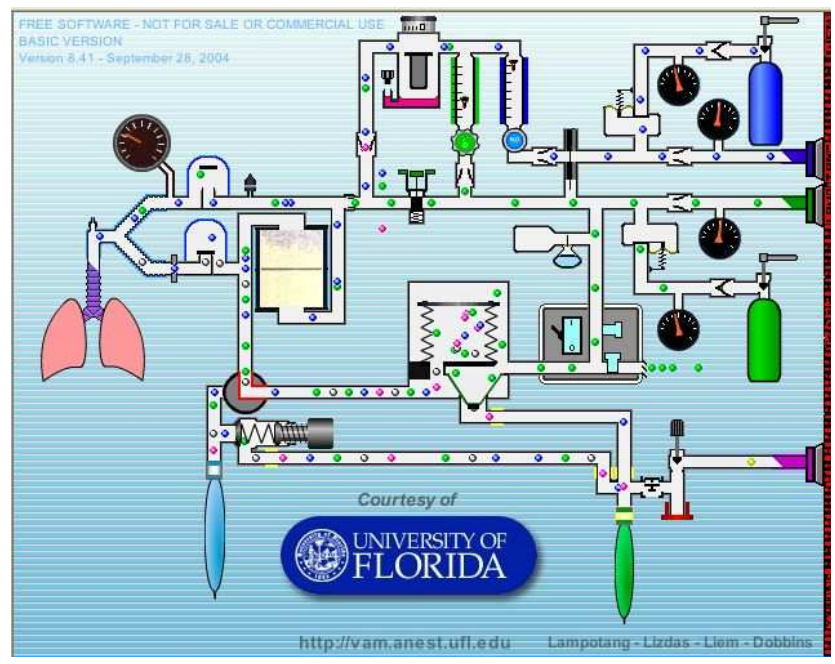

Figure 2. A screenshot from the VAM.

In previous research, the Augmented Anesthesia Machine (AAM) was presented as a potential solution to this problem. The AAM is a TUI that offers students the abilities to (1) use a tracked 6DOF magic lens (figure 1) to visualize an abstract 3D simulation of the anesthesia machine's internal components and invisible gas flow (figure 3), while (2) interacting with the real anesthesia machine as a TUI. The AAM helped users to better transfer their abstract knowledge of the machine (i.e. invisible gas flows) to a concrete domain (i.e. physical interaction with the machine) [16]. It was hypothesized that this improvement was the result of the TUI improving spatial cognition.

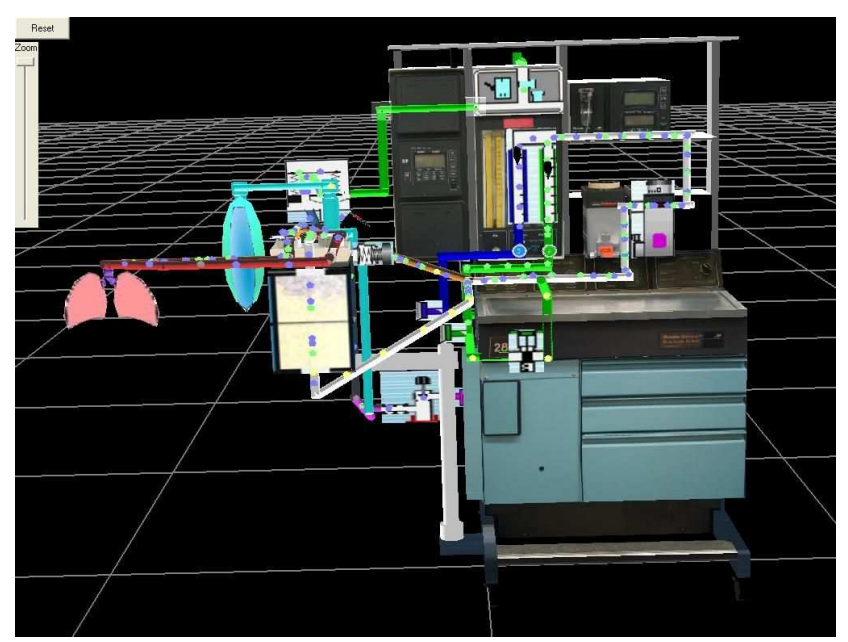

Figure 3. A zoomed out screenshot of the AAM simulation.
PreVious WorK

\subsection{Tangible User Interfaces}

A tangible interface [6] is an interface that employs real objects "as both representations and controls for computational media."'13] For example, a classic interface for a computer simulation is a Graphical User Interface (GUI) in which the user clicks on buttons and sliders etc. to control the simulation. The main purpose of a GUI is for control. Like a GUI, a tangible user interface (TUI) is used for control of the simulation, but the TUI is also an integral part of that simulation. Rather than just being a simulation control, a TUI also represents a virtual object that is part of the simulation. In this way, interacting with the real object (i.e. a real anesthesia machine) facilitates interaction with both the real world and the virtual world at the same time and in collocated space. For example, in [9], NASA engineers performed a virtual assembly using real tools in Mixed Reality (MR). Through interacting with a real tool as a tangible interface, they were able to interact with the virtual objects and complete the assembly.

One of the key aspects of our work that differentiates it from current work in TUIs is that the computational media is an abstract simulation model (i.e. VAM). This model is a complex, dynamic, network of information (i.e. gas flow). Mapping this sort of abstract model to the corresponding physical system (i.e. the AM) presents significantly greater challenges than mapping the more commonly incorporated virtual objects in MR systems (i.e. text, icons, static 3D models). For example, abstract simulation models are often simplified and spatially organized differently than the corresponding physical system. A mapping between the abstract model and the physical phenomena is often ambiguous. Much of our research focuses on general ways of using TUIs to aid in combining abstract models with the corresponding physical system.

\subsection{Magic Lens Display}

Magic Lenses were originally created as 2D interfaces, outlined in [1]. 2D magic lenses are movable, semi-transparent 'regions of interest' that show the user a different representation of the information underneath the lens. They were used for such operations as magnification, blur, and previewing various image effects. Each lens represented a specific effect. If the user wanted to combine effects, two lenses could be dragged over the same area, producing a combined effect in the overlapping areas of the lens. The overall purpose of the magic lens was to show underlying data in a different context or representation. This purpose remained when it was extended from $2 \mathrm{D}$ into $3 \mathrm{D}$ [15]. Instead of using squares and circles to affect the underlying data on a $2 \mathrm{D}$ plane, boxes and spheres were used to give an alternate visualization of volumetric data.

In Mixed and Augmented reality [11] these lenses have again been extended to become tangible user interfaces and display devices as in [10]. With an augmented reality lens, the user can look through a lens and see the real world augmented with virtual information within the lens's 'region of interest' (i.e. defined by an ARToolkit pattern marker or an LCD screen of a tablet PC based lens). The lens acts as a filter or a window for the real world and is shown in perspective with the user's first-person perspective of the real world. Thus, the MR/AR lens is similar to the original 2D magic lens metaphor, but has been implemented as a $6 \mathrm{DOF}$ tangible user interface instead of a 2D graphical user interface.

\subsection{Integrative Modeling}

Integrative modeling - the concept of linking models together in the user interface - is discussed in $[4,12]$. Our work with the AAM 
is an extension of this concept, using mixed reality to realize the linkage with an effective form of human-machine interaction. In particular, mixed reality and tangible user interfaces provide an engineering approach to blending components of the abstract VAM model with components of a real anesthesia machine.

\section{Spatial Cognition and Spatial Ability Tests}

\subsection{Working Definition}

Spatial cognition addresses how humans encode spatial information (i.e. about the position, orientation and movement of objects in the environment), and how this information is represented in memory and manipulated internally [5].

\subsection{Spatial Abilities at Different Scales}

Cognitive psychology research considers spatial cognition abilities at different scales. Each of these scales corresponds to different types of spatial challenges. For example, navigation of an environment (i.e. as in the sketch map study conducted by Billinghurst et al. [7]) would be considered large-scale, whereas the typical paper tests (i.e. the Vandenberg Mental Rotations Test) are considered small-scale tests. A person's large-scale and smallscale spatial cognition abilities may be independent

Thus, to broadly assess the spatial abilities of a person, the person should be given several tests, each of which assesses spatial ability at a different scale. For the purposes of our research, three tests are used to assess participants' spatial cognition at three different scales: figural, vista, and environmental. These spaces and the associated tests used in our study are outlined in the following sections. These tests were taken from the spatial cognition literature in psychology. For more detailed information about the tests we used, spatial ability at different scales, additional tests, and comparisons between the different tests, refer to Hegarty et al. [5].

\subsubsection{Figural: The Arrow Span Test}

The figural scale is "small in scale relative to the body and external to the individual, and can be apprehended from a single viewpoint."

To assess figural scale ability, participants in our study took the Arrow Span Test, which measures ability to maintain spatial information in working memory. The test shows participants a sequence of $2 \mathrm{D}$ arrows, shown one by one and randomly in one of 8 orientations (upright and increments of 45 degrees from upright). Participants are asked to recall the sequence from memory and type the answers using the numeric keypad. Participants are shown 15 sequences of 2D arrows. As they progress through the 15 sequences, the number of arrows in each sequence gradually increases from 2 to 6 arrows. For each arrow orientation recalled correctly, the participant gains one point. With 60 total arrows shown, there is a maximum possible score of 60 .

\subsubsection{Vista: The Perspective Taking Ability Test}

The vista scale is "projectively as large or larger than the body, but can be visually apprehended from a single place without appreciable locomotion."

To assess Vista scale ability, participants in our study took the Perspective Taking Ability Test, which "measures ability to encode, maintain, and transform spatial representations at the vista scale of space." Four objects (a cup, a keyboard, a broom, and a suitcase) were placed at the center of each wall of a real, square $8 \mathrm{~m} \times 8 \mathrm{~m}$ room. Participants were told to learn the relative locations of each of the four objects. Participants are given as much time as needed but generally do not take longer than $\sim 3$ minutes. Then, using a computer, they are asked several questions about the objects' locations. For example "You are standing in front of the cup and facing the center of the room. Point to the keyboard" The participant uses arrow keys on a keyboard to indicate the direction they are pointing. Their score is based upon how many objects are pointed to correctly and how long it takes (ms) to enter each answer.

\subsubsection{Environmental: Navigation of a Virtual Environment}

Environmental space is "large in scale relative to the body and contains the individual." Environmental tests usually include locomotion (i.e. navigating through a maze).

To assess environmental scale ability, participants navigate a virtual environment. This test assesses sense of direction. The interaction is much like a first-person-shooter video game. Participants sit at a desktop computer and use the keyboard and mouse to navigate through virtual hallways. First, participants navigate a square shaped hallway in order to learn the interface. Then they move on to a winding hallway, where there are 4 objects along the path. Participants traverse the hallway twice. On the first traversal, the objects are pointed out to the participant. On the second traversal, at each object the participant is asked to estimate distance and direction to two other objects. For direction, they point in the direction using a dial marked with 360 degrees tick marks. There are 8 distance and 8 direction estimates made in all. For distance scoring, distance estimates are correlated to actual distances. The correlation coefficient is used as the score. For directional scoring, the mean absolute difference (in degrees) of the estimated directions and actual directions is computed.

At the end of the test, participants are asked to sketch a map of the environment to scale. These 'sketch maps' are graded on a point scale. Zero is a perfect score. One point is added to the score for each object that is misplaced or left out. Additionally, one point is added to the score for each section of the path that is (a) a wrong turn, (b) an additional hallway section that does not belong, or (c) a hallway segment left out that does belong. [5]

\section{User Study: The effect of tUls on Spatial COGNITION}

\subsection{Overview}

The purpose of this study was to investigate the spatial cognition benefits of three types of interfaces: TUI, GUI, and PUI. In the study, 60 participants were trained to use an anesthesia machine using one of three interfaces (3 conditions, 20 participants per condition): (1) PUI Group - an actual anesthesia machine, (2) GUI Group - the Virtual Anesthesia Machine (VAM), or (3) TUI Group - the Augmented Anesthesia Machine (AAM). Figure 3 shows how participants progressed through the study. From our previous observations, we hypothesized that TUIs would offer more significant benefits to spatial cognition than GUIs and PUIs. Hypotheses:

H1: TUI users will have less difficulty than GUI and PUI users visualizing gas flow in the context of the real anesthesia machine.

H2: The ability of TUI users to visualize gas flow in the context of the real anesthesia machine will be less dependent on spatial ability than for GUIs and PUIs. That is, TUI training will compensate for low spatial ability.

H3: TUI users are able to understand abstract gas flow concepts, regardless of spatial ability

H4: The advantages provided by the TUI will be most directly associated with the intermediate, Vista-scale spatial ability. For the GUI and PUI groups, then, the Vista-Scale spatial 

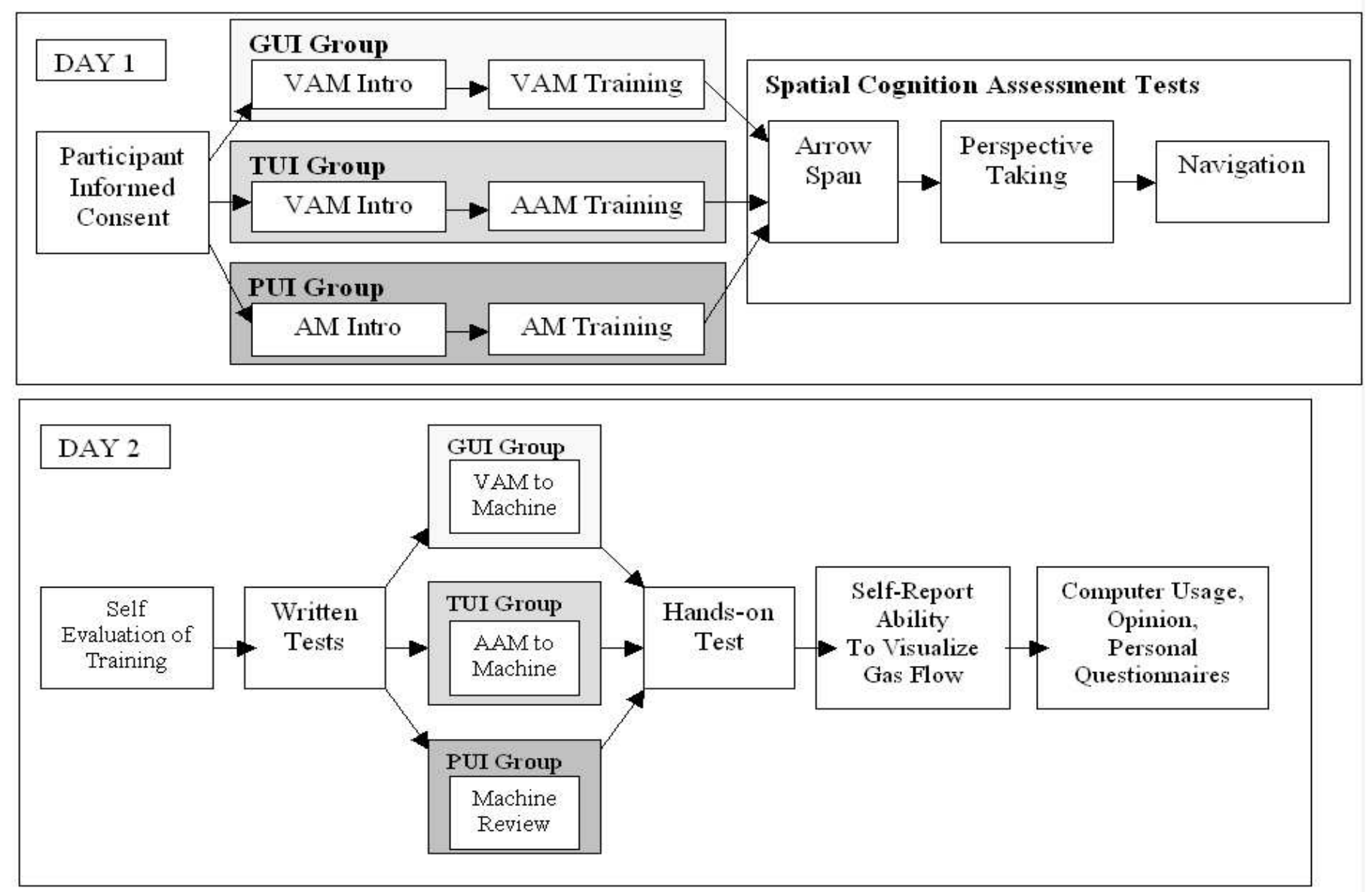

Figure 4. Study Procedure- Top: Day 1 was $\sim 90 \mathrm{~min}$. Bottom: Day 2 was $\sim 60 \mathrm{~min}$.

ability test (the Perspective-Taking Test) will be the most strongly correlated ability to performance.

\subsection{Conditions}

There were three conditions: GUI Group, TUI Group and PUI Group. The only difference between the conditions was the interface used during anesthesia machine training. These sections describe each of the interfaces in detail and the motivation for using them in the study.

\subsubsection{PUI Group: The Anesthesia Machine}

Interaction: During the training session, PUI Group interacted with the anesthesia machine only. They turned knobs, pressed buttons, and flipped switches on the anesthesia machine to change gas flow in the machine. However, unlike the other groups, participants of PUI Group had no gas flow visualization to aid them. They only had the anesthesia machine. Thus, gas flows had to be visualized mentally throughout their entire experience.

Purpose: The PUI is a physical object. It can be thought of as a TUI without any computational media. This condition is designed to show how the physical object part of a TUI affects spatial cognition.

\subsubsection{GUI Group: The Virtual Anesthesia Machine}

Interaction: During the training session, learners in GUI Group used a mouse and desktop computer to interact with the VAM (screenshot in figure 2, interaction in figure 1: GUI). The VAM is an interactive, abstract 2D transparent reality [8] simulation of the internal components and invisible gas flows of an anesthesia machine. This transparent reality approach emphasizes internal processes and structure at the expense of visual fidelity. Use of the VAM in previous studies resulted in enhanced comprehension compared to an opaque, photorealistic instantiation of an identical model of the anesthesia machine [3]. Using the mouse, users point and click on the various components to change the state of the simulation.

Purpose: The VAM is similar to the computational media part of the TUI. It is a TUI without the physical interface. Comparing the VAM to the TUI, we can investigate how the computational media part of the TUI affects spatial cognition.

\subsubsection{TUI Group: The Augmented Anesthesia Machine}

Interaction: During the training session, participants of TUI Group trained with the AAM. The AAM enables the user to interact with the real machine and visualize the effects in an overlaid simulation. To facilitate the physical interaction with the machine, the real machine's knobs and buttons are tracked using IR and color tracking coupled with common routines from the OpenCV [2] library. Then, the AAM renders spatially reorganized VAM components registered to the corresponding components of the real machine from a first person perspective. Conceptually, the VAM components are 'cut out' of the VAM and 'pasted' over the 
corresponding parts of the real machine. The collocation of the VAM components and the real anesthesia machine components demonstrates the relationships between the VAM and the anesthesia machine (figure 3).

For the visualization, the user looks through a hand-held magic lens -- a tracked 6DOF Tablet PC display (figure 5). The lens tracking system uses an outside-looking-in optical tracking technique with retro reflective markers [14]. This enables the lens to act as a see-through window into the world of the $3 \mathrm{D}$ simulation. This visualization allows the user to see how the VAM simulation flows in the context of the real machine.

The magic lens used in this study is a tablet PC (HP TC1100) It is 3.1 pounds with a 8.25 " wide, 6.25 " high display screen. The FOV was 25-90 degrees (user adjustable). Given (a) its relatively light weight and that none of the participants had complained about the weight, and (b) no two-handed interaction with the anesthesia machine would be required in the study, we opted to defer implementation of a contemplated boom arm design (which would free both of the user's hands). Moreover, none of the 60 participants complained about the magic lens occluding the view.

Purpose: A TUI combines physical objects with computational media. To understand how a TUI affects spatial cognition, it must be compared to the physical object (i.e. the anesthesia machine) and the computational media (i.e. the VAM visualization) that make up the TUI.

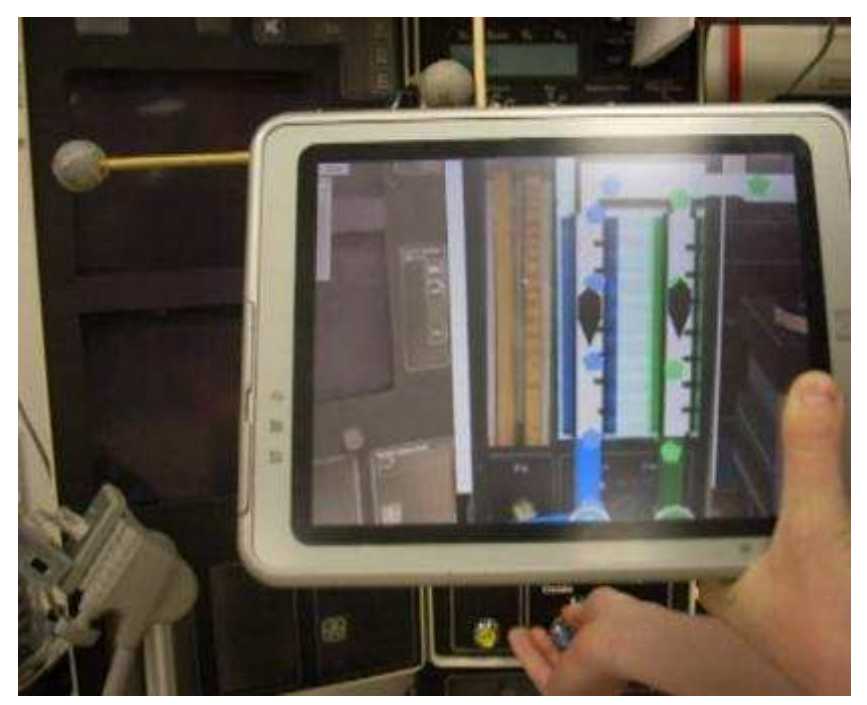

Figure 5. A participant uses the magic lens to visualize gas flow in the AAM from a first person perspective while adjusting the physical machine's nitrous oxide flow meter control knob.

\subsection{Population}

There were 60 participants in this study. All the participants were college students from several sections of an Introduction to Psychology class. All received class credit for their participation in the study. Psychology students were used because we preferred that participants had minimal prior knowledge of anesthesiology (previous knowledge could skew the data).

The study protocol was approved prior to data collection by the University of Florida IRB (\#2007-U-688), and included informed consent from the participants. Participants also had to complete a questionnaire that, among other questions, evaluated prior knowledge of anesthesia machines. No participant had prior knowledge of anesthesia machines. There were no significant differences between the semi-randomly assigned groups.

\subsection{Study Environment}

The study was conducted within one academic semester in a quiet, air-conditioned room. In each study session, there was one participant and the same investigator (to minimize interinvestigator variability) in the room for the duration of the session.

\subsection{Study Procedure}

For each participant, the study was conducted over a period of two days (figure 4). The first day included several spatial cognitive tests and the anesthesia machine training module. The second day included two tests on anesthesia machines: a written test and a hands-on test with the real machine. The second day also included several questionnaires about their opinions of the learning module and personal information (i.e. computer usage and experience, Grade Point Average - a measure of academic performance, etc). Participants were assigned semi-randomly to one of the three groups. Assignment was semi-random to keep groups the same size.

Prior to the study, participants were unaware of all the details of the study (i.e. they did not know it was about anesthesia machine training). When they arrived, they were given an informed consent form that gave them an overview of the study procedure. Participants were requested not to discuss the study with Introduction to Psychology classmates. The procedure was as follows:

\section{Day 1 ( $\sim 90$ minute session):}

1. Introduction: Once a participant finished the informed consent process they were asked to put on a white lab coat so that they would "feel more like an anesthesiologist." (The lab coat was also to reduce potential problems with the color trackers tracking their clothes.) Then they were provided a manual, which first gave them an introduction to the VAM (for groups TUI and GUI) or the AM (for PUI Group). The manual was used in conjunction with an online interactive tutorial, which highlighted and explained each of the major AM/VAM components and subsystems. The VAM was used as an intro for the TUI group because the VAM is an intrinsic component of the AAM as its computational media.

2. Relating the Introduction Material to the Anesthesia Machine: The purpose was to familiarize the participants with the parts of the real machine and how this was related to the introduction. PUI Group located all the major components on the machine. TUI Group used the magic lens to visualize each VAM icon that represented a real component underneath. GUI Group moused over each VAM component with their cursor and were shown a picture of the real component.

3. Complete 5 exercises: Each participant completed the same 5 exercises by following the manual and either interacting with a GUI (VAM), a TUI (AAM), or a PUI (AM). Each of the exercises focused on a specific anesthesia machine concept (i.e. a particular component or subsystem).

4. Spatial Cognition Tests: Participants were given three general tests to assess their spatial cognitive ability: (1) The Arrow Span Test, (2) The Perspective Taking Test and (3) Navigation of a Virtual Environment. Each of these is taken from cognitive psychology literature [5] and is outlined in section 4.

Day 2 ( $\sim 60$ minute session):

For logistical reasons, preventing participant fatigue and avoid testing superficial knowledge and short-term retention, we 
attempted to have a time interval of 24 hours between the Day 1 and Day 2 sessions.

1. Self Evaluation: Participants were asked to rate their proficiency in overall anesthesia machine understanding that was gained from the previous day.

2. Written Anesthesia Machine Test: The purpose of this test was to assess abstract knowledge gained from the previous day of training. The test consisted of short answer and multiple-choice questions from the Anesthesia Patient Safety Foundation anesthesia machine workbook [17]. Participants did not use the AM, AAM, nor the VAM to answer the questions. They could only use their machine knowledge and experience.

3. Machine Component Review: GUI Group participants were shown each 2D component in the VAM and then the investigator pointed at the real machine component. For TUI Group, the investigator pointed at each real component and the participant held the magic lens over the component to visualize the corresponding AAM component. For PUI Group, the investigator pointed out each of the main components on the real machine. No additional information was given to any of the groups. The purpose of this review was to prepare the participants for the hands-on tests that followed.

4. Hands-on Anesthesia Machine Fault Test: A 'hands-on' test was used to test participant's concrete knowledge gained from the previous day of training. For this test, participants used only the anesthesia machine without any type of computer simulation. The investigator first caused a problem with the machine (i.e. disabled a component). Then the participant had to find the problem and describe what was happening with the gas flow.

5. Self rated Gas Flow Visualization Difficulty: When participants had completed the hands-on test, the investigator explained what it meant to visualize the gas flow and how it flowed through the various components of the real machine. Participants were asked to self-rate how difficult it was to mentally visualize the gas flow in the context of the real machine on a scale of 1 (easy) to 10 (difficult).

6 Computer Usage / Personal / Opinion Questionnaires: Participants were asked several personal questions (i.e. computer experience, GPA, etc.) and what, in their opinion, the most effective and least effective parts of the training module were.

\subsection{Metrics}

The primary metrics used were the spatial cognition tests, the scores on the written tests assessing comprehension of machine function, and the self-reported ability to visualize gas flow.

Spatial Cognition Tests: Three tests were used to assess the base spatial cognitive ability of the participants. The testing procedures and scoring of the tests are described in section 4.2. These are mainly used as correlative measures to self reported ability to visualize gas flow for the different training groups.

Written Test Scores: This test included 22 short answer and 7 multiple-choice questions. It gave an overall score of a participant's abstract knowledge - the internal workings of the machine. It was rated on a 116-point scale. Each question was worth a maximum of 4 points. For the purposes of this paper, these scores were used as a correlative measure. They were used to test correlations between overall understanding of internal gas flows and spatial cognitive ability.

Self-Reported Difficulty to Visualize Gas Flow (DVGF): This is the primary metric to determine how much each interface enhanced spatial cognition. After the hands-on test, participants rated their difficulty mentally visualizing invisible gas flow in the context of the real machine. They rated the difficulty on a scale of 1 (very easy to visualize) to 10 (very difficult to visualize).

\subsection{Results and Discussion}

Note: Correlations between spatial abilities tests and DVFG are presented only for those measures that produced a significant correlation within at least one of the training groups. For Pearson correlations, the significance is marked as follows: * is $\mathrm{p}<0.10$, ** is $\mathrm{p}<0.02, * * *$ is $\mathrm{p}<0.01$.

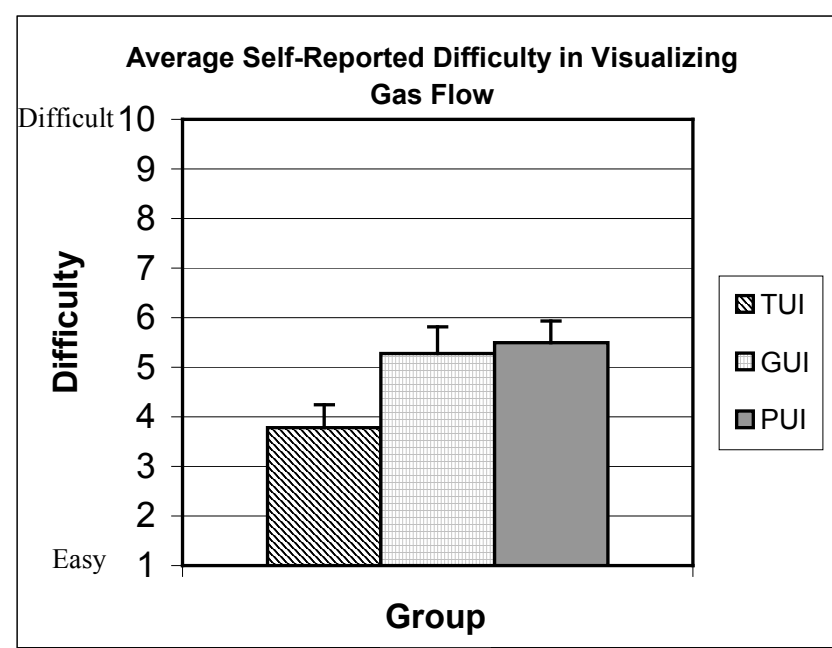

Figure 6. Average Self-Reported Difficulty in Visualizing Gas Flow in the Context of the Anesthesia Machine. 1 means very easy to visualize. 10 means very difficult to visualize. Standard error bars are shown as well.

Table 1. Self-Reported Difficulty in Visualizing Gas Flow in the Context of the Anesthesia Machine (DVGF)

\begin{tabular}{|l|l|l|}
\hline Group & Average & Stdev \\
\hline TUI & 3.79 & 1.72 \\
\hline GUI & 5.28 & 2.13 \\
\hline PUI & 5.50 & 1.91 \\
\hline
\end{tabular}

Table 2. Significant differences in DVGF between groups

\begin{tabular}{|l|l|}
\hline Groups Compared & $\mathrm{p}$ value \\
\hline TUI - PUI & $\mathrm{p}=0.011$ \\
\hline TUI - GUI & $\mathrm{p}=0.045$ \\
\hline GUI - PUI & $\mathrm{p}=0.740$ \\
\hline
\end{tabular}

Table 3. DVGF Correlations to selected Spatial Cognition Measures. (For Arrow Span and Nav. Distance, higher correlations reflect higher ability; for Nav. Sketch, higher correlations reflect lower spatial ability)

\begin{tabular}{|l|l|l|l|}
\hline Group & Arrow Span & Nav. Distance & $\begin{array}{l}\text { Nav. Sketch } \\
\text { Map }\end{array}$ \\
\hline TUI & 0.005 & -0.120 & -0.066 \\
\hline GUI & $-0.404^{*}$ & $-0.553^{* * *}$ & $0.618^{* * *}$ \\
\hline PUI & $-0.539^{* * *}$ & 0.139 & 0.161 \\
\hline
\end{tabular}

Table 4. Written Test Scores

\begin{tabular}{|l|l|l|}
\hline Group & Average Points & Stdev \\
\hline TUI & 45.4 & 18.3 \\
\hline GUI & 48.3 & 21.5 \\
\hline PUI & 43.8 & 16.2 \\
\hline
\end{tabular}


Table 5. Written Test Score correlations to spatial ability

\begin{tabular}{|l|l|l|l|}
\hline Group & Arrow Span & Nav. Distance & $\begin{array}{l}\text { Nav. Sketch } \\
\text { Map }\end{array}$ \\
\hline TUI & 0.177 & -0.111 & -0.334 \\
\hline GUI & 0.320 & 0.270 & $-0.500^{* *}$ \\
\hline PUI & $0.614^{* * *}$ & -0.012 & -0.230 \\
\hline
\end{tabular}

\subsubsection{DVGF Discussion}

The DVGF rating measured the difficulty that a participant had when visualizing gas flow in the context of the real machine layout. TUI Group DVGF scores were significantly lower (Averages: figure 6, Table 1; $\mathrm{p}$ values: Table 2) than both PUI Group and GUI Group. TUI found it significantly less difficult to mentally visualize gas flow between components of the real machine. Thus, we accept the hypothesis \#1 that TUI users have less difficulty than GUI and PUI users when visualizing gas flow in the context of the real anesthesia machine.

One problem with the DVGF is that it is self-reported and not a direct measure of actual performance. Rather, it is a measure of the user's perception of their gas flow visualization abilities. However, when used with other measures (as explored in the following sections), self-reports can be an effective way of probing spatial cognitive abilities, such as the ability to mentally visualize gas flow. In this way, the DVGF is similar to the selfreported sense of direction test found in Hegarty et al [5]. Because of this, we claim DVGF is a valid metric for measuring ability to mentally visualize gas flow.

\subsubsection{No Differences in Spatial Ability Among the Groups}

Before reporting on correlations to spatial ability it is important to note that there were no significant differences among the groups for any of the spatial cognition tests. Therefore, no group had a significant spatial cognitive advantage over another group.

\subsubsection{DVGF Correlations to Spatial Ability Discussion}

GUI Group's spatial ability test scores were significantly correlated to their DVGF scores on three of five measures. (table 3 ). This shows that GUI users with lower spatial ability had more difficulty visualizing gas flow in the context of the real machine. Because GUI users had very little exposure to the real anesthesia machine, GUI users with low spatial cognition had difficulty mentally mapping the VAM to the spatially reorganized real anesthesia machine.

Like GUI Group, PUI Group's DVGF ratings also had significant correlations to spatial ability (table 3 ) in the arrow span test. This correlation demonstrates that PUI Group participants with lower small-scale spatial ability (as assessed by the arrow span test) experienced more difficulty in visualizing gas flow.

For PUI Group, it was surprising that there were only correlations between the arrow span test and the DVGF (rather than being correlated to both the navigation tests and arrow span tests as in GUI Group). We hypothesize that this is related to the entirely different way that PUI Group approached problems with the machine. After the hands-on test, all participants were asked whether they tried to visualize the gas flow during the test. All participants in GUI Group and TUI Group, said yes. Only one participant in PUI Group said yes. PUI Group participants solved problems by only thinking about the controls - knobs, buttons etc. Perhaps this problem solving approach does not require largescale spatial ability, as measured by the navigation test.

Unlike the other groups, TUI Group's DVGF ratings showed no correlation to any of the three spatial tests (table 3). For TUI Group, then, spatial ability had no impact on difficulty of visualizing gas flow. Thus, we accept the hypothesis \#2 that TUI users were able to mentally visualize gas flow effectively, regardless of spatial ability.

\subsubsection{Written Test Correlations to Spatial Ability Discussion}

Because the DVGF was self-reported, an additional measurement was used that tested overall understanding of the machine's internal gas flows: the written test. For example, one question asked, "Is the inhalation valve bidirectional or unidirectional and why?" To correctly answer this question, one would need a deep understanding of the flow of invisible gases in the machine. This test did not measure how well participants could visualize the gas flows in the context of the machine. It only measured how well they understood the gas flows conceptually. Thus, there were few spatial mapping problems involved. Note that there were no significant differences in the written test scores between any of the three conditions (table 4). However, this conceptual understanding is likely correlated to actual gas flow visualization ability, which is why it is analyzed here as a secondary measure.

GUI Group's Written Test scores was significantly correlated with the navigation sketch map score (table 5). This correlation demonstrates that GUI Group participants with lower large-scale spatial ability had more difficulty in understanding gas flow concepts. We hypothesize that this could be related to the fact that the VAM presents the gas flow information only in a small-scale spatial layout. This could have improved their small-scale spatial cognition, but inhibited their large-scale spatial cognition.

PUI Group's test scores had a significant correlation to the Arrow Span Test (table 5). This suggests that PUI Group participants with lower small-scale spatial ability had more difficulty in understanding gas flow concepts. We hypothesize that this could be related to the more procedural problem solving approach taken by PUI Group. The procedural problems are aided by large-scale spatial ability, which may be improved by the PUI. The conceptual problems are aided by small-scale spatial ability, which could be improved by the GUI.

Unlike the other groups, TUI Group's written test scores had weak and non-significant correlations to all three of the selected spatial ability tests. This suggests that both large-scale and smallscale spatial ability had only minimal effects on participants' conceptual understanding of the internal workings of the machine. Thus, we accept hypothesis \#3: TUI users were able to understand abstract gas flow concepts effectively, regardless of spatial ability.

\subsubsection{Types of Problems in which TUls Improve Spatial Cognition}

Of the three spatial ability tests used in the study, only the Arrow Span test and Navigation test yielded significant results when correlated to other measures. The Vista-scale spatial ability scores were not correlated to written test performance nor DVGF ratings for any of the three groups. Thus, Hypothesis \#4 is rejected.

The arrow test and the navigation tests are indicative of the types of spatial problems for which TUIs are most effective. A TUI's merging of spaces (at least in the anesthesia training domain) improves two types of spatial cognition: (1) figural (i.e. the arrow test - small-scale) and (2) environmental (i.e. navigation - large-scale). Vista scale problems (as tested by the Perspective Taking Test) are relatively unaffected by TUI's merging of spaces in the anesthesia training domain.

Moreover, tests like the Arrow Span Test and the Navigation test can be used to screen for individuals who will likely experience the most significant benefits from TUIs. In accepting 
hypotheses H1, H2, and H3, we conclude that TUI users with low spatial cognition will not be impeded by their spatial ability as they would with other types of interfaces (i.e. GUI, PUI). Thus, in future studies, we will study this population (people with low spatial ability) more closely and use the Arrow Span and Navigation tests to determine which individuals are part of this population.

Spatial challenges (such as the challenges of visualizing gas flow) can be detrimental to the learning process for students with low spatial cognition. This is the problem that anesthesia educators have observed when students transfer from the VAM to the AM. This study demonstrates one of the most powerful benefits of TUIs: TUIs can help users with low spatial cognition to be less challenged by their spatial ability. This unique benefit is likely due to TUIs merging physical objects with computational media. By combining these two spaces, which are usually separated in traditional interfaces, the TUI improves the users' spatial cognition.

\section{Conclusions}

A study was conducted to determine how TUIs improve spatial cognition. In the study, there were sixty participants trained in anesthesia machine operation. Twenty participants trained using a TUI - the AAM. Twenty participants trained with a GUI - the VAM. Twenty participants trained with a PUI - the AM. By comparing how each of these interfaces affected the participants' spatial cognition, we were able to determine that the TUI compensated for low spatial cognition in anesthesia training. More generally, our results provide empirical support for what is often assumed but rarely experimentally tested - that tangible user interfaces can offer significant cognitive benefits to individuals with low spatial cognition.

Spatial ability is highly variable in the general population. This can cause many users significant difficulty in tasks that require high spatial ability. The study conducted shows that the TUI can significantly lower this variance for certain types - scales - of spatial problems. Specifically, TUIs improve spatial cognition in figural and environmental scale spatial tasks. The TUI's merging of spaces makes it easier for users to perform spatial cognition tasks. This demonstrates one of the most unique and powerful benefits of a TUI: TUIs enable users with low spatial cognition to be less challenged by spatial problems. The careful evaluation of different scales of spatial cognition, and the demonstration that they may differentially be involved in certain advantages of different kinds of interfaces, is we believe a novel and important aspect of our work.

In general, the tangible user interface in this study (the AAM) was created by mapping an abstract simulation model (the VAM) to a physical system (the AM). This type of mapping can be applied to many other types of physical systems and their corresponding simulation models. For example, if we consider the anesthesia machine as a system of prototypical "black boxes" and accept that we are surrounded by "black box" objects (printers, car engines, computers, ATM machines, DVD players, etc), then the results of this study have the potential to be relevant beyond anesthesia machines to objects in our everyday life.

\section{ACKNOWLEDGEMENTS}

This research is supported by NSF Grant IIS-0643557. A special thanks goes to David Lizdas, Cynthia Kaschub, and the study participants. Some of the technology mentioned here is UF patent pending.

\section{REFERENCES}

[1] Bier, E. A., M. C. Stone, K. Pier, W. Buxton and T. D. DeRose (1993). "Toolglass and magic lenses: the see-through interface." Proceedings of the 20th annual conference on Computer graphics and interactive techniques: $73-80$.

[2] Bradski, G. (2000). "The OpenCV Library." Dr. Dobb's Journal November 2000, Computer Security.

[3] Fischler, I., Kaschub, C. E., Lizdas, D. E., Lampotang, S. (2007). "Understanding of Anesthesia Machine Function is Enhanced with a Transparent Reality Simulation." submitted to Simulation in Health Care

[4] Fishwick, P. A. (2004). "Toward an Integrative Multimodeling Interface: A Human-Computer Interface Approach to Interrelating Model Structures." SIMULATION 80(9): 421.

[5] Hegarty, M., D. R. Montello, A. E. Richardson, T. Ishikawa and K. Lovelace (2006). "Spatial abilities at different scales: Individual differences in aptitude-test performance and spatial-layout learning." Intelligence 34(2): 151-176.

[6] Ishii, H. and B. Ullmer (1997). "Tangible bits: towards seamless interfaces between people, bits and atoms." Proceedings of the SIGCHI conference on Human factors in computing systems: 234241.

[7] Billinghurst, M. and Weghorst, S. (1995). "The use of sketch maps to measure cognitive maps of virtual environments." In Proceedings of the Virtual Reality Annual international Symposium (Vrais'95) (March 11 - 15, 1995).

[8] Lampotang, S., D. E. Lizdas, N. Gravenstein and E. B. Liem (2006). "Transparent reality, a simulation based on interactive dynamic graphical models emphasizing visualization." Educational Technology 46(1): 55-59.

[9] Lok, B. C. (2004). "Toward the merging of real and virtual spaces." Communications of the ACM 47(8): 48-53.

[10] Looser, J., M. Billinghurst and A. Cockburn (2004). "Through the looking glass: the use of lenses as an interface tool for Augmented Reality interfaces." Proceedings of the 2nd international conference on Computer graphics and interactive techniques in Australasia and South East Asia: 204-211.

[11] Milgram, P. and F. Kishino (1994). "A Taxonomy of Mixed Reality Visual Displays." IEICE Transactions on Information Systems 77: 1321-1329.

[12] Park, M. and P. A. Fishwick (2005). "Integrating Dynamic and Geometry Model Components through Ontology-Based Inference." SIMULATION 81(12): 795.

[13] Ullmer, B. and H. Ishii (2000). "Emerging frameworks for tangible user interfaces." IBM Systems Journal 39(3): 915-931.

[14] Van Rhijn, A. and J. D. Mulder (2005). "Optical Tracking and Calibration of Tangible Interaction Devices." Proceedings of the Immersive Projection Technology and Virtual Environments Workshop 2005.

[15] Viega, J., M. J. Conway, G. Williams and R. Pausch (1996). "3D magic lenses." Proceedings of the 9th annual ACM symposium on User interface software and technology: 51-58.

[16] Quarles, J., S. Lampotang, I. Fischler, P. Fishwick, and B. Lok, (2008) "A Mixed Reality Approach for Merging Abstract and Concrete Knowledge" to appear in the Proceedings of IEEE Virtual Reality 2008.

[17] Lampotang, S., D.E. Lizdas, E.B. Liem, J.S. Gravenstein (2007): The Anesthesia Patient Safety Foundation Anesthesia MachineWorkbook v1.1a. Retrieved December 25, 2007, from University of Florida Department of Anesthesiology Virtual Anesthesia Machine Web site: http://vam.anest.ufl.edu/members/workbook/apsf-workbookenglish.html 